\title{
Lógica Fuzzy e Processo Analítico Hierárquico (AHP) aplicados ao Zoneamento de Áreas Suscetíveis a Deslizamentos: Uma Revisão
}

\author{
Fuzzy Logic and Analytic Hierarchy Process (AHP) applied to Zoning of Areas \\ Susceptible to Landslides: A Review
}

Celso Henrique Leite Silva Junior ${ }^{1}$

\begin{abstract}
${ }^{1}$ Engenheiro Ambiental, Divisão de Sensoriamento Remoto - DSR, Instituto Nacional de Pesquisas Espaciais - INPE, São José
\end{abstract} dos Campos - SP, Brasil.

\section{Resumo}

\begin{abstract}
A ação do homem sobre a natureza tem se destacado nas últimas décadas. Devido a essa ação, são observados diversos impactos no ambiente, principalmente os decorrentes do aumento populacional e da ocupação de áreas irregulares, que vão desde os ligados ao saneamento básico até aos desastres naturais. Nesse sentido, o presente artigo teve como objetivo apresentar um panorama do uso da Lógica Fuzzy e do Processo Analítico Hierárquico - AHP para o zoneamento de áreas suscetíveis a deslizamentos. Nesse panorama são descritas as duas ferramentas, são apresentados os principais trabalhos realizados no Brasil e no exterior além da definição de termos. Essas ferramentas se demonstram eficientes e úteis do ponto de vista metodológico, uma vez que vários trabalhos já comprovaram a sua eficácia, onde se destacam os operadores Fuzzy Média Ponderada AHP e Gamma, além da concreta eficiência no uso do Processo Analítico Hierárquico - AHP com o objetivo de tratar as incertezas inerentes ao uso do conhecimento empírico de especialista.
\end{abstract}

Palavras-chave: Desastres Naturais. Meio Ambiente. Impacto Ambiental. Mapeamento de Deslizamentos.

\begin{abstract}
The action of man over nature has been highlighted in recent decades. Due to this action, many impacts on the environment are observed, mainly arising from population growth and irregular occupation of areas, ranging from those related to basic sanitation by the natural disasters. In this sense, this paper aims to present an overview of the use of Fuzzy Logic and the Analytic Hierarchy Process - AHP for zoning areas susceptible to landslides. In this overview we describe the two tools are presented the main works in Brazil and abroad and the definition of terms. These tools are demonstrated efficient and useful methodological point of view, since several studies have demonstrated its effectiveness, with the focus on Fuzzy Weighted Average AHP and Gamma operators, beyond the specific efficiency in the use of the Analytic Hierarchy Process - AHP with designed to address the inherent uncertainty of the empirical use of expert knowledge.
\end{abstract}

Keywords: Natural Disasters. Environment. Environmental Impact. Landslide Mapping. 


\section{Introdução}

A questão ambiental tem se destacado nas últimas décadas na sociedade devido aos resultados da ação do homem sobre a natureza. Os impactos ambientais e sociais, observados na atualidade são os decorrentes da ocupação urbana de forma desordenada que trazem consigo problemas, como a deficiência no saneamento básico, além de problemas como os alagamentos e deslizamentos quando da ocupação de áreas irregulares.

No Brasil, nas últimas décadas, observou-se um intenso processo de urbanização da população, onde o último senso populacional mostrou que $84 \%$ (160.925.804 de habitantes) da população brasileira vive em áreas urbanas (IBGE, 2010). Dessa maneira observa-se uma tendência da população em viver em áreas urbanas. Muitas das vezes essas áreas ocupadas não apresentam condições de infraestrutura mínima para sustentar a população, causando problemas sociais, ambientais e além dos associados à segurança da população.

Essa concentração da população nos grandes centros urbanos não tem sido acompanhada de ações governamentais eficientes para o ordenamento do uso e ocupação do solo, isso tem levado principalmente a população mais carente a ocupar áreas naturalmente inadequadas ou, suscetíveis a eventos naturais, onde são ocupadas sem os mínimos preceitos técnicos e observância da legislação vigente (PFALTZGRAFF, 2007).

Nesse contexto surge a preocupação com a ocorrência de desastres naturais que podem ser definidos como o resultado do impacto de fenômenos naturais extremos ou intensos sobre um sistema social, que causa sérios danos e prejuízos que excede a capacidade da comunidade ou da sociedade atingida em conviver com o impacto (MARCELINO, 2008).

No ano de 2012 os desastres naturais tiveram um impacto significativo na sociedade brasileira, oficialmente foi relatada a ocorrência de 376 desastres naturais, os quais causaram 93 óbitos e afetaram cerca de 16.977.614 pessoas (BRASIL, 2013). Desse total, cerca de 26 óbitos foram causados por deslizamentos, onde cerca de 123.555 pessoas foram afetadas. Dos desastres que causaram mortes à população brasileira, os deslizamentos corresponderam por cerca de $27.96 \%$ dos óbitos (BRASIL, 2013). Nesse cenário surge a necessidade de ferramentas que possibilitem estudar de maneira integrada esses fenômenos.

No contexto atual, devido a acessibilidade a dados ambientais espacializados, é possível a sua integração em Sistemas de Informação Geográfica (SIG) por meio da aplicação de técnicas de Inferência Espacial. Segundo Meirelles et al. (2007a), de maneira geral os modelos de inferência mais utilizados são: operadores booleanos, sobreposição por índice ou média ponderada com pesos e Lógica Fuzzy. O mesmo autor constatou que dos métodos de inferência geográfica realizados em SIG, os baseados na Lógica Fuzzy obtiveram os melhores resultados na realização de diversos estudos ambientais.

No cenário do acelerado crescimento populacional no Brasil, que vem acompanhado da ocupação de áreas ambientalmente irregulares, e a necessidade de identificação de áreas suscetíveis (áreas com maior ou menor probabilidade de ocorrência) a deslizamentos, o presente artigo teve como objetivo apresentar um panorama do uso da Lógica Fuzzy e do Processo Analítico Hierárquico - AHP para o zoneamento de áreas suscetíveis a deslizamento. 


\section{Zoneamento, Suscetibilidade e Deslizamento}

\subsection{Zoneamento}

Com base na recomendação da Associação Brasileira de Geologia de Engenharia e Ambiental (ABGE), zoneamento é "a divisão do solo em áreas ou domínios homogêneos e sua classificação de acordo com graus de suscetibilidade, perigo ou risco de deslizamentos reais ou potenciais, ou ainda de acordo com a aplicabilidade de regulamentações ligadas ao perigo" (FELL et al., 2008, p. 86).

Silva e Santos (2011), também definem o zoneamento como o ato de dividir uma área em setores (zonas), a partir de critérios pré-determinados, onde cada zona deve estar sujeita a normas específicas que podem enfocar uma linha de ação onde cada zona tem um grau de similaridade e relação entre os elementos componentes, e uma diferença claramente identificada entre as zonas vizinhas.

Dessa maneira, cada unidade zoneada tem alto grau de associação dentro de si, com as variáveis envolvidas solidamente ligadas, com significativa diferença entre ela e os outros compartimentos, concluindo-se assim, que o zoneamento faz uma análise por agrupamentos passíveis de serem desenhados no eixo horizontal de uma determinada área e numa escala específica (SANTOS, 2004).

O zoneamento é o resultado de um processo que envolve diversos passos, como a definição de indicadores, o levantamento de dados sobre os indicadores, a elaboração integrada das informações que conduz ao diagnóstico, e à definição de limites que definem as zonas (SILVA; SANTOS, 2011).

\subsection{Suscetibilidade}

A suscetibilidade deve ser entendida como a probabilidade de ocorrência de um deslizamento decorrente das condições do terreno, ou seja, é avaliada com base na correlação espacial entre fatores de predisposição como declividade, geologia cobertura e uso do solo e etc. (MENESES, 2011). Zêzere (2007, p. 17 apud MENESES, 2011, p. 15) também define suscetibilidade como a "propensão do território à ocorrência de um tipo particular de deslizamento, com base nos fatores condicionantes da instabilidade".

Com base na recomendação da ABGE, a suscetibilidade a deslizamento pode ser definida também como, "uma avaliação quantitativa ou qualitativa do tipo, do volume ou área, e da distribuição espacial dos deslizamentos que existem ou potencialmente possam ocorrer em uma área. A suscetibilidade também pode incluir uma descrição da velocidade e da intensidade do deslizamento, potencial ou existente" (FELL et al., 2008, p. 97).

\subsection{Deslizamento}

"Geólogos, engenheiros e outros profissionais sempre se apoiam em definições de deslizamentos específicas e com ligeira diferença entre si. Essa diversidade de definições reflete a complexidade da natureza das muitas disciplinas associadas ao estudo desse fenômeno" (HIGHLAND; BOBROWSKY, 2008, p. 6).

Seguindo recomendação da ABGE considera-se deslizamento como "o movimento da massa de rochas, colúvios ou terra (solo) que desliza em uma encosta" (FELL et al., 2008, p. 86). "Existem outros termos que são usados alternativamente ao termo "deslizamento", que incluem movimento de massa, ruptura do talude, etc." (HIGHLAND; BOBROWSKY, 2008, p. 6).

Fell et al. (2008) comenta que o termo "deslizamento" se refere tanto aos deslizamentos que já existem, os considerados deslizamentos conhecidos, quanto a deslizamentos potenciais, os quais podese obter a probabilidade de ocorrência com base na geologia, geometria e processos de formação de encostas. 


\section{Classificação dos Deslizamentos}

Tendo em vista a classificação dos tipos de deslizamentos, essas estão associadas a mecanismos específicos de falhas em taludes e às propriedades e características desses tipos de falhas geológicas.

Segundo Highland e Bobrowsky (2008, p. 7): o tipo de movimento descreve a mecânica interna de como a massa é deslocada: queda, envergamento, escorregamento, espalhamento ou escoamento. Assim, os deslizamentos são descritos pelo uso de dois termos que se referem respectivamente, ao tipo de movimento e ao material (ou seja, queda de rocha, de detritos, etc.). Deslizamentos também podem formar uma ruptura complexa, que pode incluir mais de um tipo de movimento (ou seja, deslizamento de rocha e fluxo de detritos).

Os deslizamentos podem ocorrer praticamente em qualquer lugar do mundo, do ponto de vista tradicional de que deslizamentos estão restringidos a áreas extremamente íngremes e terrenos inóspitos não reflete adequadamente a natureza real do problema. De alguma maneira, a maioria dos países do planeta já foi afetada por esse tipo de fenômeno. A razão para tal amplitude geográfica está muito relacionada à existência de vários mecanismos desencadeadores. Chuvas em excesso, terremotos, vulcões, incêndios florestais e outros mecanismos recentes, como certas atividades humanas perigosas, são apenas algumas das causas chaves que podem provocar um deslizamento (HIGHLAND; BOBROWSKY, 2008).

A classificação a seguir dos tipos de deslizamentos foi extraída da publicação do Banco Mundial (World Bank) intitulada "The landslide handbook: a guide to understanding landslides" de autoria de Highland e Bobrowsky (2008). Essa classificação consolida os principais tipos de deslizamentos que ocorrem ao longo do globo, podendo ser utilizada em qualquer localidade para classificar esses fenômenos.

Quedas, se inicia com a separação do solo ou da rocha, ou de ambos, de um talude íngreme, ao longo de sua superfície, na qual tenha ocorrido pouco ou nenhum deslocamento por cisalhamento. Posteriormente, o material vem abaixo, principalmente por queda, salto ou rolamento. As Quedas se dividem em dois tipos, a Queda rochosa (Figura 1A e 1B) e o Tombamento (Figura 1C e 1D).

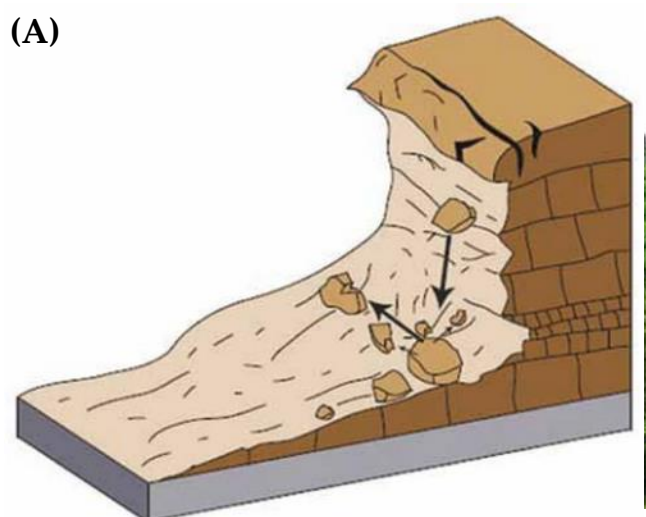

(B)
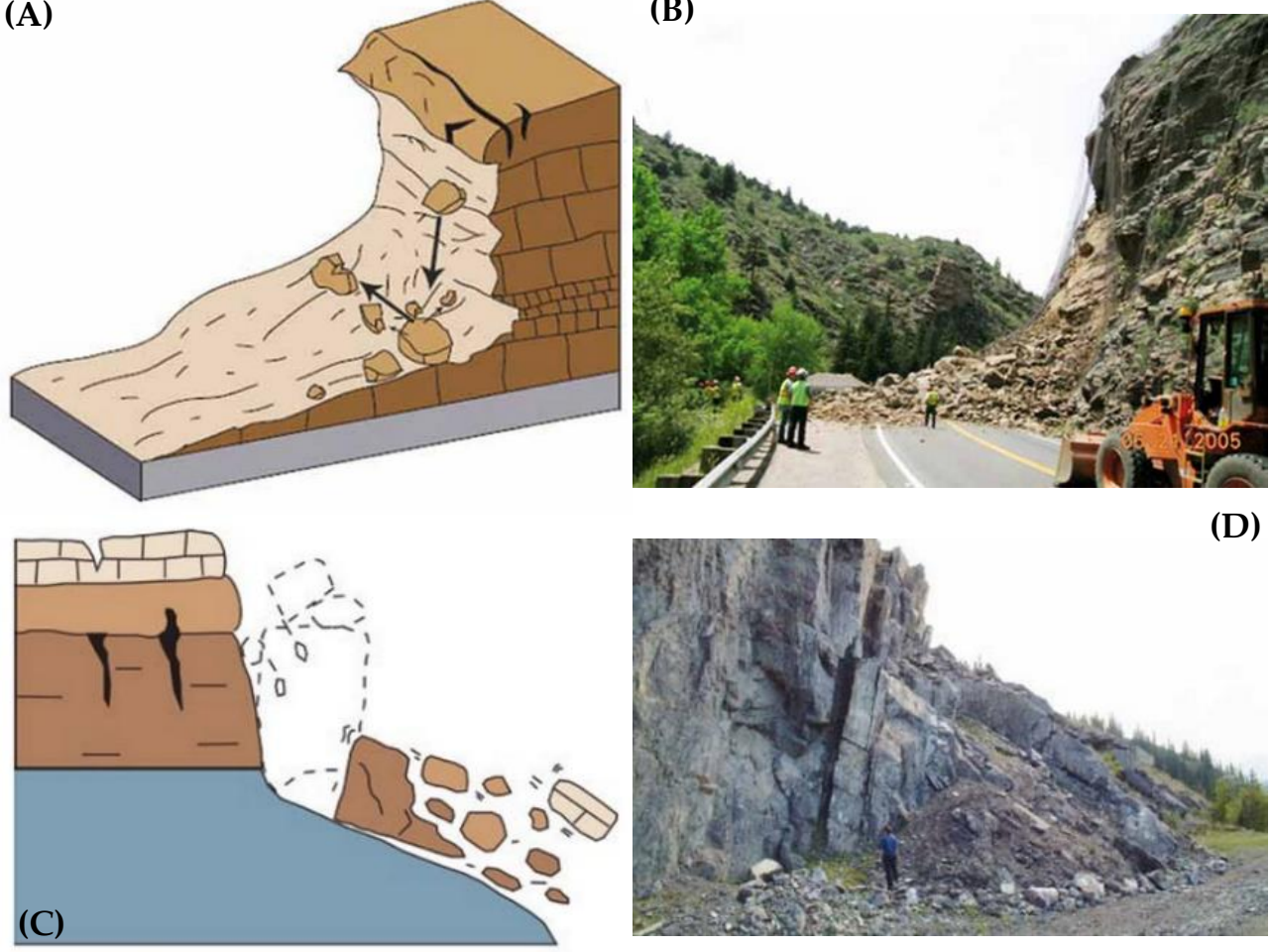

Figura 1 - (A) Esquema de queda rochosa. (B) Exemplo de queda rochosa (Foto por Colorado Geological Survey). (C) Esquema de tombamento. (D) Exemplo de tombamento (Foto por G. Bianchi Fasani). Fonte: Highland e Bobrowsky (2008). 
Escorregamentos, são um movimento de uma massa de solo ou rocha, em um declive, que ocorre sobre superfícies em ruptura ou sobre zonas relativamente finas com intensa deformação por cisalhamento, esse movimento não ocorre inicialmente de maneira simultânea, por toda área que vem a se tornar a superfície da ruptura; o volume de material deslocado aumenta a partir de uma área no local da ruptura. Podem ser rotacionais (Figura 2A e 2B) ou translacionais (Figura 2C e 2D).
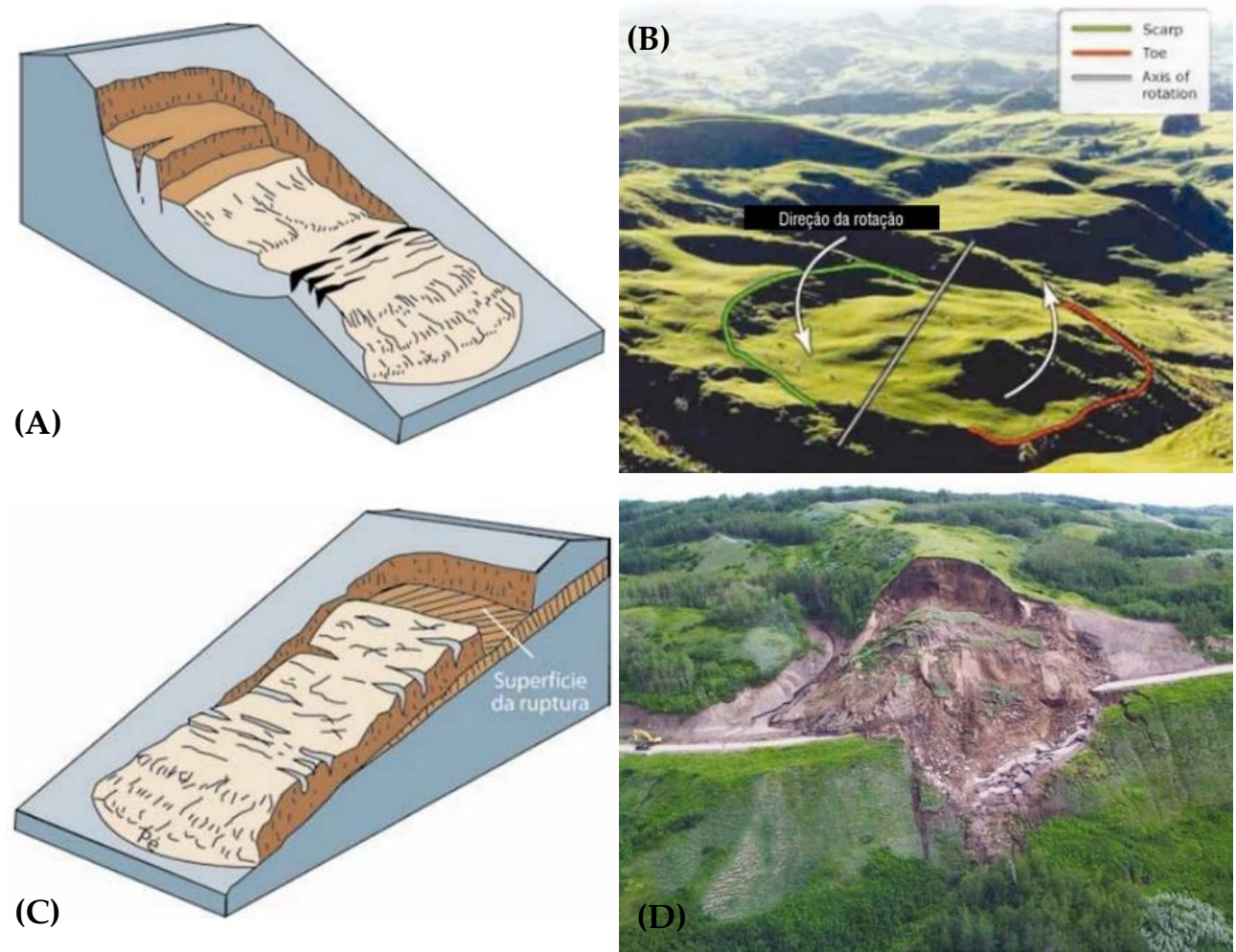

Figura 2 - (A) Esquema de escorregamento rotacional. (B) Exemplo de escorregamento rotacional ((Foto por Michael J. Crozier, Encyclopedia of New Zealand, atualizada em 21 de setembro de 2007). (C) Esquema de escorregamento translacional. (D) Exemplo de escorregamento translacional ((Foto por Réjean Couture, Canada Geological Survey). Fonte: Highland e Bobrowsky (2008).

Espalhamento, é uma extensão de uma massa coesiva, formada por solo ou rocha, combinada a um generalizado afundamento da superfície da massa fraturada de material coesivo para uma camada inferior, de material menos rígido. Espalhamentos podem ser o resultado de liquefação ou fluxo (extrusão) do material menos rígido. Tipos de espalhamentos podem ser em blocos, por liquefação e laterais. Tendo como único tipo o espalhamento lateral (Figuras 3A e 3B).

(A)

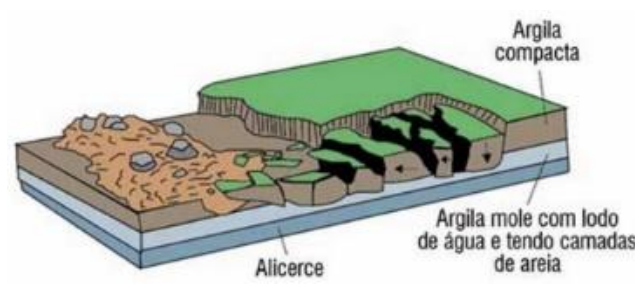

(B)

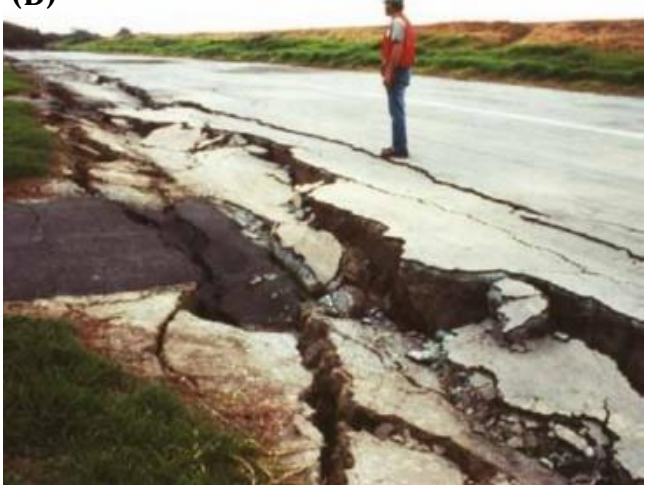

Figura 3 - (A) Esquema de espalhamento lateral (uma camada passível de liquefação está abaixo da camada superficial). (B) Exemplo de espalhamento lateral (Foto por Steve Ellen, U.S. Geological Survey).

Fonte: Highland e Bobrowsky (2008). 
Escoamento, é um deslizamento contínuo espacialmente em que as superfícies de cisalhamento têm vida curta, espaço reduzido entre si e não são preservadas, seus componentes de velocidade na massa que se desloca em um escoamento são semelhantes às de um líquido viscoso, observa-se com frequência, uma mudança gradativa de escorregamentos para escoamentos, dependendo da quantidade de água presente, da mobilidade e da evolução do movimento. Os escoamentos podem ser divididos em Fluxos de detritos (Figuras 4A e 4B), Fluxos de detritos vulcânicos (Lahars) (Figuras 4C e 4D), Avalanche de detritos (Figuras 4E e 4F), Fluxo de terra (Figuras 5A e 5B), Fluxos lentos de terra (Figuras 5C e 5D) e Escoamentos em subsolos permanentemente gelados (Figuras 5E e 5F).
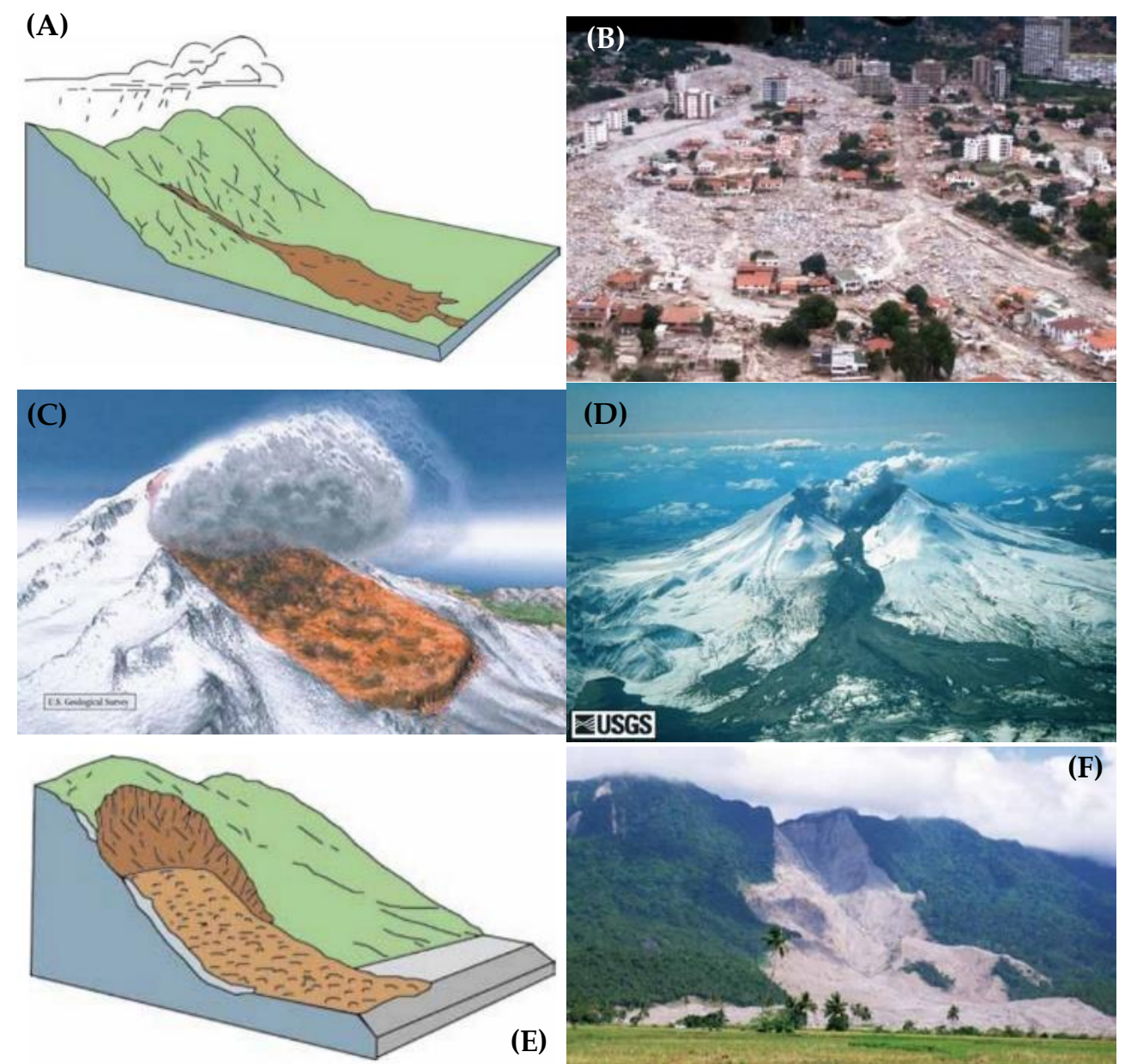

Figura 4 - (A) Esquema de fluxo de detritos. (B). Exemplo de fluxo de detritos (Foto por L. M. Smith, Waterways Experiment Station, U.S. Army Corps of Engineers). (C) Esquema de um lahar. (D) Exemplo de um lahar (Foto por Tom Casadevall do U. S. Geological Survey). (E) Esquema de avalanche de detritos. (F) Exemplo de avalanche de detritos (Foto da Equipe Geotécnica da Universidade de Tóquio).

Fonte: Highland e Bobrowsky (2008). 

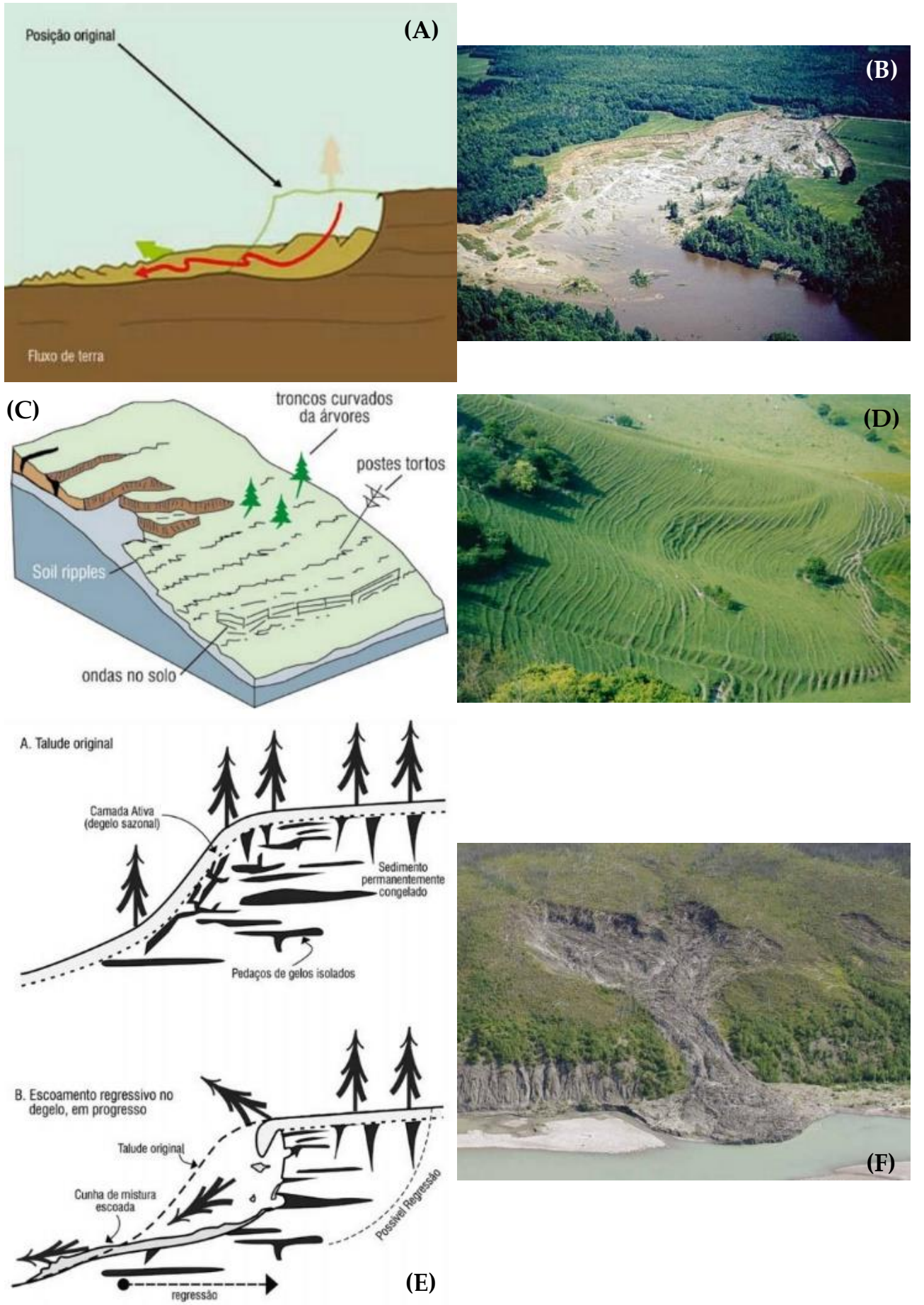

Figura 5 - (A) Esquema de um fluxo de terra. (B) Exemplo de um fluxo de terra (Foto de G. R. Brooks do Geological Survey of Canada). (C) Esquema de um deslizamento lento de terra (rastejo). (D) Exemplo de um deslizamento lento de terra (Foto de Ian Alexander). (E) Esquema de um escoamento regressivo no degelo (Esquema por Jan Aylsworth, Geological Survey of Canada). (F) Exemplo de um escoamento regressivo no degelo (Foto de Marten Geertsema, Ministry of Forests, British Columbia, Canadá).

Fonte: Highland e Bobrowsky (2008). 


\section{Lógica Fuzzy}

\subsection{Conjuntos Fuzzy}

O conjunto Fuzzy foi introduzida no contexto científico por Zadeh (1965), através da publicação do artigo intitulado "Fuzzy Sets" no Journal Information and Control (JANÉ, 2004).

$O$ conjunto de valores fuzzy $A$ em $X$ é definido matematicamente como o par ordenado: $A=\{(x$, $\left.\mu_{\mathrm{A}}(\mathrm{x})\right\}, x \in X$, em que $X=(\mathrm{x})$ é o universo de atributos, $\mu_{\mathrm{A}}(\mathrm{x})$ é conhecido como o grau de pertinência de $x$ em A. $O$ valor $\mu_{\mathrm{A}}(\mathrm{x})$ é um número que pertence ao intervalo $[0,1]$, onde 1 representa a associação total do conjunto, e 0 a não-associação (MEIRELLES et al., 2007b; WANG et al., 2009; ZADEH, 1965).

Os graus de associações de $\mathrm{x}$ em A refletem uma espécie de ordem que não é baseada na probabilidade, mas na possibilidade. Estes Valores são obtidos por funções de pertinência (MEIRELLES et al., 2007b; WANG et al., 2009; ZADEH, 1965): $\mu_{\mathrm{A}}(\mathrm{x}): \mathrm{X} \rightarrow \mathrm{A}:[0,1]$.

A seguintes notações são utilizadas para representar os conjuntos fuzzy: $A=\int \mathrm{x} \mu \mathrm{A}(\mathrm{x}) / \mathrm{x}$, quando A é contínuo ou $\mathrm{A}=\sum \mu_{\mathrm{Aj}}(\mathrm{x}) / \mathrm{xj}$, ou seja, $\mu_{\mathrm{A} 1}(x) / x_{1}+\mu_{\mathrm{A} 2}(x) / x_{2}+\cdots+\mu_{\mathrm{An}}(x) / x_{n^{\prime}}$ quando A é finito por um conjunto contável de n elementos (MEIRELLES et al., 2007b; WANG et al., 2009; ZADEH, 1965).

"O símbolo '/ deve ser interpretado como com respeito a e ' $x$ ' como união" (MEIRELLES et al., 2007b, p. 126).

\subsection{Teoria Fuzzy}

“Também conhecida como lógica nebulosa ou difusa" (AGUADO; CANTANHEDE, 2010, p. 1), essa teoria tem como principal objetivo lidar com dados que contém algum tipo de incerteza. Ao usar a teoria difusa, cada objeto ou declaração é dado em um valor no intervalo entre 0 e 1 , indicando a sua adesão a um determinado conjunto. Cada objeto pode ser membro de vários grupos com diferentes valores de adesão. Este conceito é muito útil para a categorização de dados e para a tomada de decisão, ao contrário da lógica booleana que produz resultados com respostas rígidas (MARJANOVIC; CAHA, 2011).

A ideia da Teoria Fuzzy, não apenas fica restrita entre verdadeiro e falso, mas sim existem vários níveis entre o verdadeiro e falso. De modo figurativo enquanto a lógica clássica enxerga apenas o preto e o branco, a lógica fuzzy é capaz de além do preto e do branco, enxergar vários tons de cinza (KOHAGURA, 2007) (Figura 6).

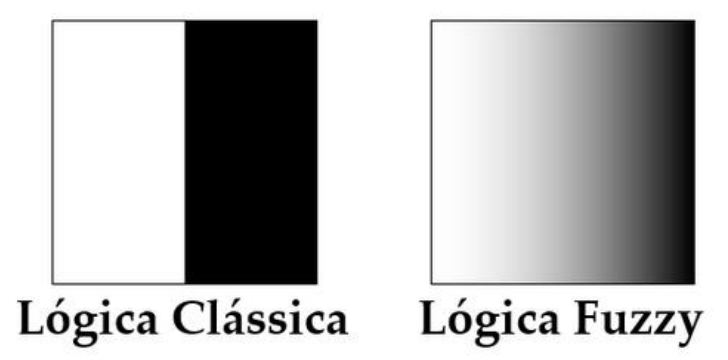

Figura 6 - Comparativo entre a Lógica Clássica e a Logica Fuzzy.

Fonte: Adaptado de Kohagura (2007).

Nesse sentido, a Teoria Fuzzy cria linguisticamente escalas de valores que buscam quantificar o objeto a ser estudado. O uso dessa teoria implica na criação das chamadas variáveis linguísticas que, diferentemente da lógica clássica, são de extrema relevância pois possibilitam captar o grau de incerteza presente nessas variáveis e traduzir para um modelamento matemático. Esta possibilidade da lógica fuzzy é chamada grau de participação, ou também conhecido como função de pertinência (AGUADO; CANTANHEDE, 2010). 


\subsection{Estrutura do Sistema Lógico Fuzzy}

A estrutura de todo o sistema lógico fuzzy está baseada em três operações que estão explicitadas na Figura 7.

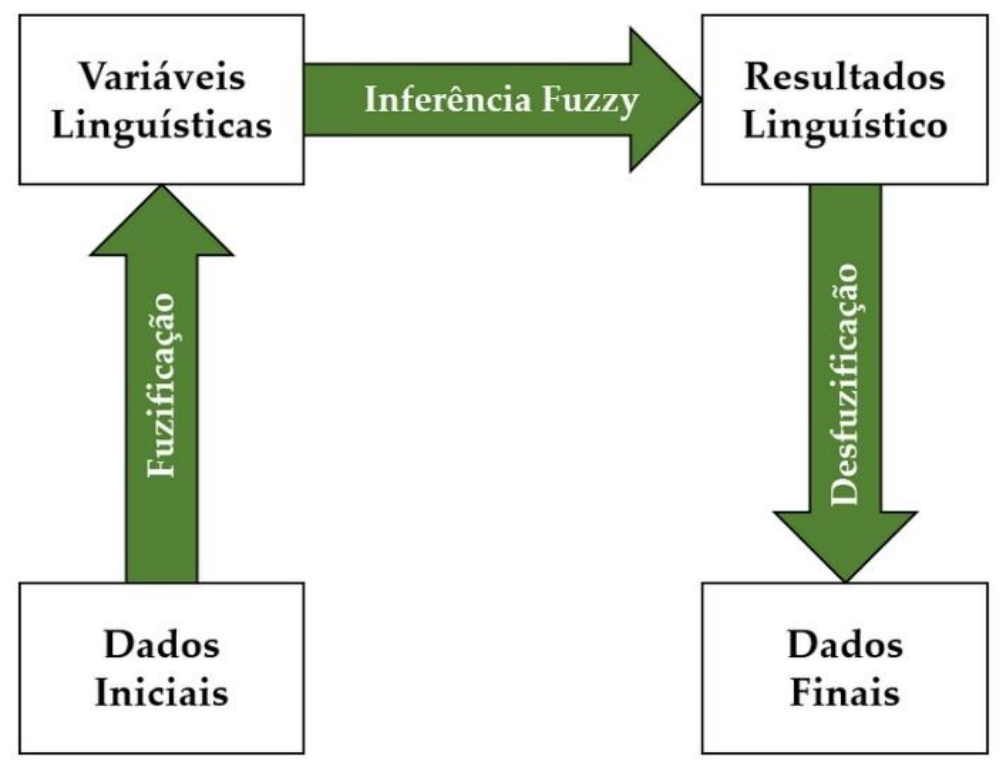

Figura 7 - Sistema Lógico Fuzzy.

Fonte: Adaptado de Cox (1995).

Na etapa de fuzificação ocorre a transformação dos dados de entrada iniciais em suas respectivas variáveis linguísticas, nesta etapa, todas as informações relativas à imprecisão ou incerteza associada a estas variáveis devem ser consideradas, e deve ser considerada a consulta a especialistas da área estudada para a atribuição de valores relacionados aos graus de pertinência para cada uma das variáveis em estudo, contribuindo assim para maior precisão nos resultados ou o uso de conhecimento já estabelecido sobre a área (JANÉ, 2004).

Na etapa de inferência Fuzzy cuja finalidade é relacionar as possíveis variáveis entre si, através de regras pré-estabelecidas, cumprindo assim com os objetivos do algoritmo, algoritmos esses também denominados operadores Fuzzy (JANÉ, 2004).

A terceira e última etapa do sistema lógico fuzzy é chamada desfuzificação, e de acordo com VonAltrock (1996), consiste na tradução do resultado linguístico do processo de inferência fuzzy, em um valor numérico (processo de fatiamento), entretanto Cox (1995), apresenta um outro conceito onde diz que compreende o processo de conversão de um número fuzzy em um número real.

\subsection{Operadores Fuzzy}

Existem vários operadores fuzzy para a combinação de funções de pertinência. Os operadores mais conhecidos são: o Produto Algébrico, Soma Algébrica, Operador Gamma e Média Ponderada AHP (MARJANOVI; CAHA, 2011; MEIRELLES et al., 2007b).

O operador Produto Algébrico e dado por (Equação 1):

$$
U_{i}=\prod_{i=1}^{n} W_{i}
$$

Onde $W_{i}$ é o valor de pertinência fuzzy para cada mapa que deverão ser combinados, $U_{i}$ é o valor do pixel resultante. Os resultados tendem a ser muito pequenos quando se utiliza este operador, devido ao efeito de se multiplicar diversos números menores do que 1 (CÂMARA et al., 2004). 
Soma Algébrica - "Este operador é complementar ao produto Algébrico Fuzzy, e é representado por" (CÂMARA et al., 2004, p. 11) (Equação 2):

$$
U_{i}=1-\prod_{i=1}^{n} W_{i}\left(1-W_{i}\right)
$$

"O resultado desta operação é sempre um valor maior ou igual ao maior valor de pertinência fuzzy de entrada. O efeito desta operação é, portanto, de aumentar o valor de pertinência" (CÂMARA et al., 2004, p. 11).

Observando-se a equação, conclui-se que enquanto o Produto Algébrico é um produto algébrico, a soma algébrica Fuzzy, não é uma simples soma algébrica (ESCADA, 1998).

"O operador Gamma inclui o produto entre a soma algébrica Fuzzy e produto Fuzzy, e é representado por" (ESCADA, 1998, p. 9; KAYASTHA et al., 2013a; MARJANOVI; CAHA, 2011) (Equação 3):

$$
U_{i}=\left(1-\prod_{i=1}^{n} W_{i}\left(1-W_{i}\right)\right)^{\gamma}\left(\prod_{i=1}^{n} W_{i}\right)^{1-\gamma}
$$

Onde $\gamma$ é o valor gamma que varia de 0 a $1, W_{i}$ é o valor de pertinência fuzzy para cada mapa que deverão ser combinados. "No operador gamma pode-se variar a importância de cada termo (soma algébrica e produto algébrico Fuzzy). A importância de cada termo no operador gama é definida atribuindo-se valores entre (0,1) para o expoente $\gamma^{\prime \prime}$ (CÂMARA et al., 2004, p. 11).

Esse operador executa a multiplicação dos membros dos diferentes mapas, sendo que o valor de saída de um dado ponto é sempre menor ou igual ao valor do menor membro Fuzzy. Isto ocorre devido a multiplicação de valores iguais ou menores que 1 (CÂMARA et al., 2004).

Quando o valor gamma for igual a 0 , o resultado será igual ao produto algébrico, e quando igual a 1, o resultado será igual à soma algébrica. Os valores de gamma entre 0 e 0,35 apresentaram um caráter "diminutivo", ou seja, sempre menor ou igual ao menor membro fuzzy de entrada. Valores gamma entre 0,8 e 1,0 terão um caráter "aumentativo" onde o valor de saída será igual, ou maior que o valor do maior membro Fuzzy de entrada. Os valores de gama entre 0,35 e 0,8 não apresentaram resultados nem de caráter "aumentativo" nem "diminutivo", os valores de saída, cairão sempre entre o menor e o maior valor dos de entrada (BONHAM-CARTER, 1994 apud CÂMARA et al., 2004). A Figura 8 demonstra de forma ilustrativa o comportamento desse operador.

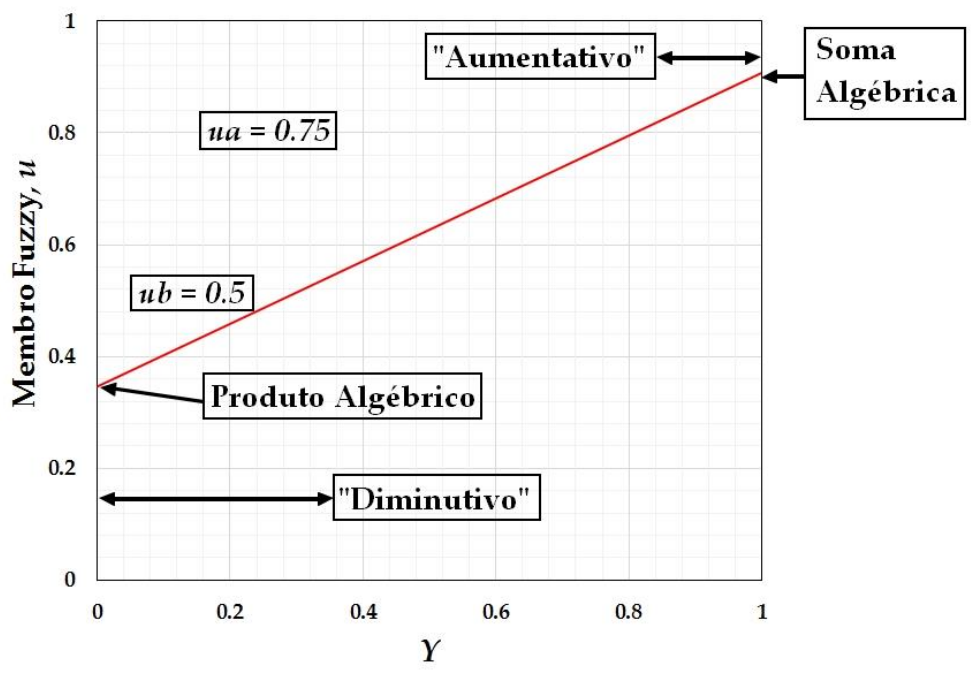

Figura 8 - Gráfico de distribuição da função Fuzzy Gamma.

Fonte: Modificado de Câmara et al. (2004, p. 13). 
No operador Média Ponderada AHP, os pesos de cada membro Fuzzy de entrada (evidência) assim como o valor de ponderação são definidos segundo a técnica de tomada de decisão AHP (CÂMARA et al., 2004; SAATY, 1980). A equação desse operador é obtida da equação da média ponderada.

A equação do algoritmo Média Ponderada AHP é descrita da seguinte maneira (Equação 4):

$$
U_{i}=\sum_{i=1}^{n} w_{i} x_{i} / \sum_{i=1}^{n} x_{i}
$$

Onde $w_{i}$ é o valor do peso da evidência de entrada calculada a partir do uso do AHP, e $x_{i}$ é o de entrada da evidência que pode ser obtida a partir de métodos empíricos com a ajuda de especialista, ou ainda com o uso do empirismo com auxílio do AHP (CÂMARA et al., 2004).

De acordo com Meirelles et al. (2007b), na comparação de vários operadores fuzzy com o objetivo de realizar mapeamento a respeito de pesquisas minerais, concluíram que o operador Média Ponderada AHP apresentou os melhores resultados de acurácia através da validação dos dados em campo.

\section{Processo Analítico Hierárquico - AHP}

O Processo Analítico Hierárquico, do inglês Analytic Hierarchy Process - AHP, foi desenvolvido por Saaty (1980). “O AHP é uma metodologia matemática destinada a ponderar quantitativamente variáveis mediante a interação do pesquisador com o modelo matemático e fazer isso em forma de considerações qualitativas" (RAFFO, 2012, p. 26).

O processo AHP envolve a identificação de um problema de decisão e, em seguida, decompõe este em uma hierarquia de "subproblemas" menores e mais simples, onde cada um poderia então ser analisado de forma independente, sem perder o foco do problema de decisão (SOUZA et al., 2013). Os pesos são atribuídos aos critérios, de acordo com sua importância relativa de acordo com a Tabela 1. As alternativas são avaliadas com base nesta importância relativa, por meio de comparações de pares, usando a escala de Saaty de números absolutos onde se atribui valores numéricos tanto quantitativos, como qualitativos aos julgamentos (SOUZA et al., 2013).

Tabela 1 - Definição e explicação dos 9 pesos fundamentais de julgamento comparativo. Fonte: Pinese Júnior e Rodrigues (2012, p. 9).

\begin{tabular}{ccc}
\hline $\begin{array}{c}\text { Intensidade de } \\
\text { Importância }\end{array}$ & Definição & Explicação \\
\hline 1 & Importância Equiparada & $\begin{array}{c}\text { Contribuição equiparada para o } \\
\text { objetivo. }\end{array}$ \\
3 & Importância Moderada & $\begin{array}{c}\text { Um objetivo ligeiramente favorável em } \\
\text { detrimento de outro }\end{array}$ \\
5 & Importância Forte & $\begin{array}{c}\text { Um objetivo fortemente favorável em } \\
\text { detrimento de outro. }\end{array}$ \\
7 & Importância Muito Forte & $\begin{array}{c}\text { Um objetivo muito fortemente favorável } \\
\text { em detrimento de outro; domínio } \\
\text { demonstrado na prática. }\end{array}$ \\
9 & Importância Absoluta & $\begin{array}{c}\text { É a maior ordem de afirmação possível } \\
\text { de um objetivo em detrimento de outro. } \\
\text { Possível necessidade de se interpolar } \\
\text { julgamentos numéricos. }\end{array}$ \\
\hline
\end{tabular}


Uma vez construída a hierarquia, avalia-se sistematicamente seus elementos, comparando-os um ao outro, em pares. Ao fazer as comparações, utilizam-se dados concretos sobre os elementos, ou julgamentos sobre o significado relativo ou a importância dos elementos (SHAHABI et al., 2014). O AHP converte os julgamentos em valores numéricos que podem ser processados e comparados sobre toda a extensão do problema. Um peso numérico, ou prioridade, é derivado para cada elemento da hierarquia, permitindo que elementos distintos e frequentemente incomensuráveis sejam comparados entre si (SHAHABI et al., 2014).

As comparações entre os atributos e as alternativas são registradas em matrizes na forma de frações entre 1/9 e 9. Cada matriz é avaliada pelo seu autovalor para verificar a coerência dos julgamentos. Este procedimento gera uma "razão de coerência" que será igual a 1 se todos os julgamentos forem coerentes entre se mesmos (Figura 9) (PINESE JÚNIOR; RODRIGUES, 2012).

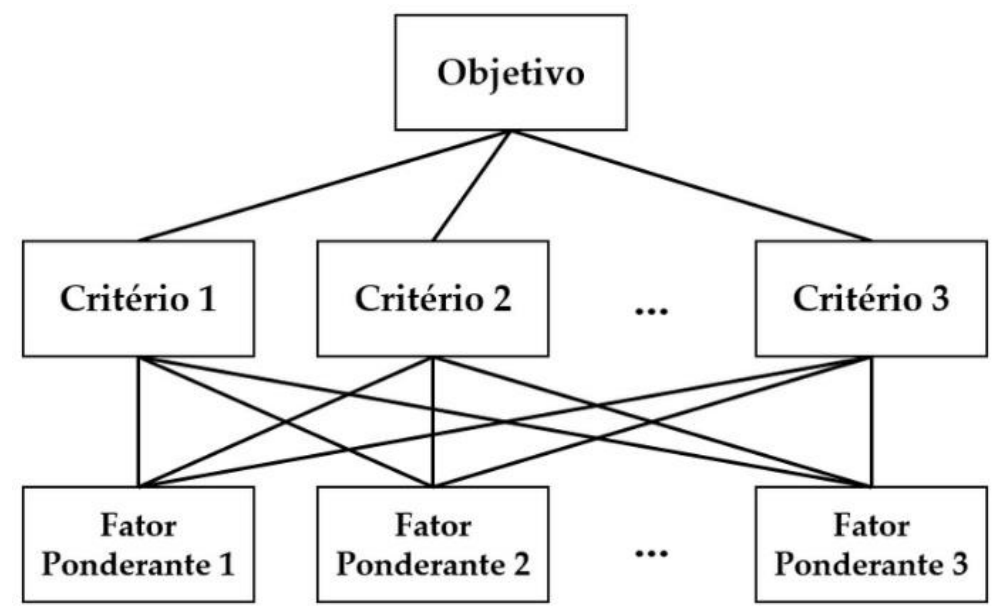

Figura 9 - Modelo simplificado e esquemático de árvore hierárquica.

Fonte: Adaptado de Carvalho e Mingoti (2005) apud Pinese Júnior e Rodrigues (2012, p. 10).

Existem vários instrumentos disponíveis na rede mundial de computadores que facilitam o uso do AHP, onde essa ferramenta já está implementada, e possui uma interface gráfica de usuário amigável que facilita o seu uso para os mais diversos fins.

Segundo Raffo (2012) Os procedimentos matemáticos da metodologia AHP podem ser implementados usando uma calculadora eletrônica, um software do tipo planilha eletrônica como o Excel da Microsoft, mas nestes casos o usuário deve conhecer bastante dos fundamentos teóricos da metodologia, no entanto, existem programas de computador onde a metodologia já está implementada e o usuário só deve conversar com o sistema, exemplos disso são os softwares WebHIPRE (WEB-HIPRE, 2014), o programa Spring (CÂMARA et al., 1996; INPE, 2014) e o PriEsT (SIRAJ et al., 2013).

Para um aprofundamento sobre os fundamentos teóricos da metodologia, recomenda-se a leitura do artigo elaborado por Pinese Júnior e Rodrigues (2012) intitulado "O método de análise hierárquica - AHP - como auxílio na determinação da vulnerabilidade ambiental da bacia hidrográfica do Rio Piedade (MG)" publicado na Revista do Departamento de Geografia da Universidade de São Paulo (USP).

\section{Zoneamento de Áreas Suscetíveis a Deslizamentos}

Atualmente, devido a acessibilidade a dados ambientais espacializados, é possível a sua integração em Sistemas de Informação Geográfica (SIG) através da aplicação de técnicas de Inferência Espacial. Segundo Meirelles et al. (2007b), de maneira geral os modelos de inferência mais utilizados são: operadores booleanos, sobreposição por índice ou média ponderada com pesos e Lógica Fuzzy. Meirelles et al. (2007b) constatou que dos métodos de inferência geográfica realizados em SIG os 
baseados na Lógica Fuzzy obtiveram os melhores resultados na realização de diversos estudos ambientais.

Nos últimos anos a utilização dos produtos de sensoriamento remoto integrados a SIGs para análises ambientais tem se demostrado uma importante ferramenta. Diversos estudos podem ser realizados através da análise espacial integrada em ambientes SIG. Dentre esses estudos destaca-se as análises e mapeamento, ou zoneamento de áreas suscetíveis a deslizamento.

Muitas pesquisas vêm sendo realizadas no Brasil e em outros países, tanto na análise e/ou mapeamento de áreas suscetíveis a deslizamento. Dentre essas pesquisas destacam-se os estudos realizados no Brasil por: Cristo (2002), que analisou a suscetibilidade a riscos naturais relacionados a enchentes e deslizamentos no setor leste da bacia hidrográfica do Rio Itacorubi em Florianópolis - SC, Dias e Herrmann (2002), que realizaram um estudo de caso a respeito da suscetibilidade a deslizamentos no bairro Saco Grande em Florianópolis - SC, Marcelino (2004), que realizou mapeamento de áreas suscetíveis a escorregamento no município de Caraguatatuba - SP utilizando técnicas de sensoriamento remoto, Muñoz (2005), que realizou análise comparativa entre técnicas de inferência espacial para identificação de áreas suscetíveis aos movimentos de massa na região de São Sebastião - SP, Pfaltzgraff (2007), que realizou mapeamento de suscetibilidade a deslizamentos na região metropolitana do Recife, Vestena (2010) mapeou a susceptibilidade a deslizamentos na bacia hidrográfica de Caeté, município de Alfredo Wagner/SC, região serrana de Santa Catarina, utilizando o modelo SINMAP (Stability Index MAPping), para determinar o índice de estabilidade das vertentes, a partir da combinação de um modelo hidrológico com um de estabilidade de encostas., Bispo et al. (2011) analisaram a suscetibilidade aos movimentos de massa em São Sebastião - SP com o uso de métodos de inferência espacial, Moro et al. (2011) realizou levantamento de suscetibilidade a deslizamento e erosão em encostas no Morro da Cruz na cidade de Itajaí - SC, Vanacôr e Rolim (2012), que realizaram mapeamento da suscetibilidade a deslizamentos utilizando técnicas de estatística bivariada e sistema de informação geográfica no nordeste do Rio Grande do Sul e Canavesi et al. (2013) analisaram a suscetibilidade a deslizamentos de terra no município de Paraibuna - SP. Também no Brasil Silva Junior et al. (2013; 2014), realizaram o zoneamento de áreas suscetíveis a deslizamentos na bacia hidrográfica do Rio Anil no município de São Luís - MA, utilizando vários operadores Fuzzy, com auxílio do Processo Analítico Hierárquico - AHP, além de ter realizado validação das áreas identificadas utilizando dados de campo e Índice Kappa.

$\mathrm{Na}$ Colômbia destaca-se o trabalho realizado por Delgado (2006) que utilizou sistema de informação geográfica para avaliação da suscetibilidade a deslizamentos em uma região do eixo cafeteiro colombiano. Lopes (2008), avaliou regionalmente a suscetibilidade a deslizamentos no Concelho de Santarém em Portugal. Pereira (2009), analisou o perigo a movimentos de vertentes na região norte de Portugal. Guilard e Zêzere (2010) realizaram avaliação e validação da suscetibilidade a deslizamentos no Concelho de Loures em Portugal. Meneses (2011) analisou a suscetibilidade e o risco a movimentos de vertente no Concelho de Tarouca em Portugal. Piedade et al. (2011) analisou modelos de suscetibilidade a deslizamentos superficiais na região norte de Lisboa em Portugal. Na bacia do Rio Safarood no Iran, Pourghasemi et al. (2012) mapeou áreas suscetíveis a deslizamento de terra usando índices de modelos de probabilidade e entropia condicional em sistema de informação geográfica, em Jinbu na Coréia do Sul, Lee et al. (2013) aplicaram dados orientados a funções de validação para realizar mapeamento da suscetibilidade a deslizamento de terra, Ozdemir e Altural (2013) realizaram um estudo comparativo da relação entre frequência, pesos de evidência e métodos de regressão logística para o mapeamento as suscetibilidade a deslizamento de terra nas Montanhas Sultan no Sudoeste da Turquia, Kayastha et al. (2013) realizaram mapeamento de áreas suscetíveis a deslizamento de terra usando a abordagem da Lógica Fuzzy na área de Ghurmi-Dhad Khola, na parte oriental do Nepal, Conforti et al. (2014) realizou avaliação da capacidade de redes neurais artificiais para o mapeamento de suscetibilidade a deslizamento de terra na bacia do rio Turbolo no norte da Calábria na Itália.

Recomenda-se a leitura da publicação da Associação Brasileira de Geologia de Engenharia e Ambiental (ABGE) intitulada "Diretrizes para o zoneamento da suscetibilidade, perigo e risco de deslizamentos para planejamento do uso do solo" (MACEDO; BRESSANI, 2013), publicação traduzida 
a partir do artigo "Guidelines for landslide susceptibility, hazard and risk zoning for land use planning", publicado na revista Engineering Geology (FELL et al., 2008). Nessa publicação é possível por exemplo, encontrar recomendações quanto aos tipos e níveis de zoneamento e escalas dos mapas, além de outras recomendações uteis.

\section{Considerações Finais}

É importante observar que ao longo dos últimos anos o uso da Lógica Fuzzy e do Processo Analítico Hierárquico - AHP, tem se demostrado eficiente para a integração de vários dados ambientais além do tratamento das incertezas inerentes a participação e dinâmica de cada componente ambiental, dessa maneira o seu uso torna-se importante, pois são ferramentas de fácil manipulação e de extensa bibliografia disponível. O seu uso é importante para o planejamento e para a gestão dos deslizamentos, que muitas das vezes causam perdas tanto de vidas quanto de materiais, ocasionando transtornos à sociedade.

De maneira geral os operadores Fuzzy que mais se demostraram eficientes na maioria dos trabalhos aqui citados, foram o Fuzzy Gamma e o Fuzzy Média Ponderada AHP, esse último ainda pouco usado, porem os primeiros trabalhos demonstram sua eficiência, também sendo possível ser observada no uso em outros estudos ambientais. No operador Gamma, o valor gama varia conforme a localidade de estudo, sendo necessário dessa maneira o teste de vários valores na elaboração de qualquer trabalho com o objetivo de se obter um resultado mais apurado.

É importante também observar a contribuição do AHP no tratamento das incertezas nesses tipos de trabalhos, uma vez que esses usam conhecimentos empíricos de especialistas. A Lógica Fuzzy tem uma importante contribuição no processamento e integração dos dados ambientais através do uso dos seus operadores.

O zoneamento da suscetibilidade se demonstra uma eficiente ferramenta para o planejamento e gestão dos deslizamentos, com o objetivo de evitar a perda de vidas e de materiais. Nesse contexto é importante a elaboração desse tipo de mapeamento para os municípios brasileiros como parte das ferramentas indispensáveis aos órgãos responsáveis pela gestão e monitoramento de deslizamentos.

O presente trabalho apresentou um embasamento inicial para que qualquer pesquisador que queira desenvolver trabalhos nessa temática. A revisão aqui abordada apresentou um norte de partida para a elaboração metodológica, sendo nesse sentido necessário a avaliação do pesquisador quanto ao uso dos operadores e dados ambientais a serem adotados, além da base empírica de conhecimento de especialistas a ser usada quando se fizer necessário.

\section{Referências}

AGUADO, A. G.; CANTANHEDE, M. A. Lógica Fuzzy. 2010. Disponível em: $<$ http://www.ft.unicamp.br/liag/wp/monografias/monografias/2010_IA_FT_UNICAMP_logicaFuzz i.pdf $>$. Acesso em: 20 fev. 2014.

BISPO, P. DA C. et al. Análise da suscetibilidade aos movimentos de massa em São Sebastião (SP) com o uso de métodos de inferência espacial. Geociências, v. 30, n. 3, p. 467-478, 2011.

BRASIL. Anuário Brasileiro de Desastres Naturais: 2012. 2. ed. Brasília: CENAD, 2013.

CÂMARA, G. et al. Spring: Integrating remote sensing and gis by object-oriented data modelling. Computers \& Graphics, v. 20, n. 3, p. 395-403, maio 1996. Disponível em: <http://linkinghub.elsevier.com/retrieve/pii/0097849396000088>. Acesso em: 27 jan. 2014.

CÂMARA, G. et al. Técnicas de Inferência Geográfica. In: DRUCK, S. et al. (Org.). . Análise Espacial de Dados Geográficos. Brasília: EMBRAPA, 2004. p. 250. Disponível em: $<$ http://www.dpi.inpe.br/gilberto/livro/analise/>. Acesso em: 20 fev. 2014. 
CANAVESI, V. et al. Análise da susceptibilidade a deslizamentos de terra: estudo de caso de Paraibuna, SP. 2013, Foz do Iguaçu: INPE, 2013. p. 5251-5258.

CONFORTI, M. et al. Evaluation of prediction capability of the artificial neural networks for mapping landslide susceptibility in the Turbolo River catchment (northern Calabria, Italy). CATENA, v. 113, p. 236-250, fev. 2014. Disponível em: <http://linkinghub.elsevier.com/retrieve/pii/S0341816213001963>. Acesso em: 24 mar. 2014.

COX, E. Fuzzy logic for business and industry. Massachusetts: Charles River Media Inc, 1995.

CRISTO, S. S. V. DE. Análise de susceptibilidade a riscos naturais relacionados às enchentes e deslizamentos do setor leste da bacia hidrográfica do Rio Itacorubi, Florianópolis - SC. 2002. 195 f. Dissertação (Mestrado em Geografia), Departamento de Geociências da Universidade Federal de Santa Catarina, Florianópolis - SC, 2002.

DELGADO, J. A. C. Avaliação da suscetibilidade a deslizamentos or meio do uso de sistema de informações geográficas: aplicação em uma área do eixo cafeteiro colombiano. 2006. 109 f. Dissertação (Mestrado em Geotecnia), Faculdade de Tecnologia da Universidade de Brasília, Brasília - DF, 2006.

DIAS, F. P.; HERRMANN, M. L. DE P. Susceptibilidade a deslizamentos : estudo de caso no bairro Saco Grande, Florianópolis - SC. Revista Caminhos de Geografia, v. 3, n. 6, p. 57-73, 2002.

ESCADA, M. I. S. Aplicação de Técnica Fuzzy em SIG como alternativa para o Zoneamento Ecológico Econômico (ZEE). $1998 . \quad$ Disponível em: <http://www.dpi.inpe.br/cursos/ser301/trabalhos/isabel_zeefuzzy.pdf\%E2\%80\%8E>. Acesso em: 20 fev. 2014.

FELL, R. et al. Guidelines for landslide susceptibility, hazard and risk zoning for land use planning. Engineering Geology, v. 102, n. 3-4, p. 85-98, dez. 2008. Disponível em: <http://dx.doi.org/10.1016/j.enggeo.2008.03.022>. Acesso em: 4 maio 2014.

GUILLARD, C.; ZÊEZERE, J. L. Avaliação e validação da susceptibilidade a deslizamentos no âmbito do planeamento municipal: Caso do estudo do Concelho de Loures. 2010, Porto: [s.n.], 2010. p. $273-276$.

HIGHLAND, L. M.; BOBROWSKY, P. The landslide handbook: a guide to understanding landslides. Reston: U.S. Geological Survey Circular 1325, 2008.

IBGE - Instituto Brasileiro de Geografia e Estatística. Censo Demográfico 2010. Disponível em: <http://www.sidra.ibge.gov.br>. Acesso em: 17 jan. 2014.

INPE. Spring. Disponível em: <http://www.dpi.inpe.br/spring/portugues/index.html>. Acesso em: 10 fev. 2014.

JANÉ, D. DE A. Uma introdução ao estudo da lógica fuzzy. Revista de Humanidades e Ciências Sociais Aplicadas, n. 2, p. 1-16, 2004.

KAYASTHA, P. et al. GIS based landslide susceptibility mapping using a fuzzy logic approach: A case study from Ghurmi-Dhad Khola area, Eastern Nepal. Journal of the Geological Society of India, v. 82, n. 3, p. 249-261, 15 set. 2013. Disponível em: <http://link.springer.com/10.1007/s12594-013-0147-y>. Acesso em: 4 maio 2014.

KOHAGURA, T. Lógica Fuzzy e suas aplicações. 2007. 49 f. Monografia (Graduação em Ciência da Computação), Departamento de Computação da Universidade Estadual de Londrina, Londrina - PR, 2007.

LEE, S. et al. Application of data-driven evidential belief functions to landslide susceptibility mapping in Jinbu, Korea. CATENA, v. 100, p. 15-30, jan. 2013. Disponível em: <http://linkinghub.elsevier.com/retrieve/pii/S0341816212001555>. Acesso em: 29 abr. 2014. 
LOPES, P. S. P. R. Avaliação Regional da Susceptibilidade a Deslizamentos no Concelho de Santarém. 2008. 120 f. Dissertação (Mestrado em Geografia), Departamento de Geografia da Universidade de Lisboa, Lisboa, 2008.

MACEDO, E. S. DE; BRESSANI, L. A. Diretrizes para o zoneamento da suscetibilidade, perigo e risco de deslizamentos para planejamento do uso do solo. São Paulo: ABGE/ABMS, 2013.

MARCELINO, E. V. Desastres naturais e geotecnologias: conceitos básicos. Santa Maria: CRS/INPE, 2008. Disponível em: <http://mtc-m18.sid.inpe.br/col/sid.inpe.br/mtcm18@80/2008/07.02.16.22/doc/publicacao.pdf>. Acesso em: 20 fev. 2014.

MARCELINO, E. V. Mapeamento de áreas susceptíveis a escorregamento no município de Caraguatatuba (SP) usando técnicas de sensoriamento remoto. 2004. Dissertação (Mestrado em Sensoriamento Remoto), Instituto Nacional de Pesquisas Espaciais, São José dos Campos - SP, 2004.

MARJANOVIC, M.; CAHA, J. Fuzzy approach to to landslide susceptibility zonation Fuzzy Approach Landslide Susceptibility Zonation. 2011, Pisek: [s.n.], 2011. p. 181-195. Disponível em: <http://ceurws.org/Vol-706/papersg01.pdf>.

MEIRELLES, M. S. P.; MOREIRA, F. R.; CÂMARA, G.; NETTO, A. L. C.; et al. Métodos de Inferência Geográfica: Aplicação no planejamento regional, na avaliação ambiental e na pesquisa mineral. In: MEIRELLES, M. S. P. et al. (Org.). . Geomática: Modelos e Aplicações Ambientais. Brasília: Embrapa Informação Tecnológica, 2007. p. 593.

MEIRELLES, M. S. P.; MOREIRA, F. R.; CÂMARA, G.; OUTROS. Técnicas de inferência espacial. In: MEIRELLES, M. S. P. et al. (Org.). . Geomática: Modelos e Aplicações Ambientais. Brasília: Embrapa Informação Tecnológica, 2007. p. 593.

MENESES, B. M. DO C. S. Susceptibilidade e risco de movimentos de vertente no concelho de Tarouca. 2011. 115 f. Dissertação (Mestrado em Geografia Física e Ordenamento do Território), Instituto de Geografia e Ordenamento do Território da Universidade de Lisboa, Lisboa, 2011.

MORO, G. T. et al. Levantamento de susceptibilidade a deslizamento e erosão em encostas - estudo de caso: Morro da Cruz - Itajaí - SC. 2011, Curitiba: INPE, 2011. p. 4162-4169.

MUÑOZ, V. A. Análise comparativa de técnicas de inferência espacial para identificação de unidades de suscetibilidade aos movimentos de massa na região de São Sebastião, São Paulo, Brasil. 2005. 61 f. Monografia (Especialização em Sensoriamento Remoto e Sistema de Informação Geográfica), Instituto Nacional de Pesquisas Espaciais, São José dos Campos - SP, 2005.

OZDEMIR, A.; ALTURAL, T. A comparative study of frequency ratio, weights of evidence and logistic regression methods for landslide susceptibility mapping: Sultan Mountains, SW Turkey. Journal of Asian Earth Sciences, v. 64, p. 180-197, mar. 2013. Disponível em: <http://linkinghub.elsevier.com/retrieve/pii/S1367912012005585>. Acesso em: 5 fev. 2014.

PEREIRA, S. DA S. Perigosidade a movimentos de vertente na região norte de Portugal. 2009. $370 \mathrm{f}$. Tese (Doutorado em Geografia Física), Departamento de Geografia da Universidade do Porto, Porto, 2009.

PFALTZGRAFF, P. A. D. S. Mapa de suscetibilidade a deslizamentos na região metropolitana do Recife. 2007. 151 f. Tese (Doutorado em Geologia), Centro de Tecnologia e Geociências da Universidade Federal de Pernambuco, Recife - PB, 2007.

PIEDADE, A. et al. Modelos de susceptibilidade a deslizamentos superficiais translacionais na região a norte de Lisboa. Finisterra, v. XLVI, n. 91, p. 9-26, 2011.

PINESE JÚNIOR, J. F.; RODRIGUES, S. C. O método de análise hierárquica - AHP - como auxílio na determinação da vulnerabilidade ambiental da bacia hidrográfica do Rio Piedade (MG). Revista do Departamento de Geografia - USP, v. 23, n. 2012, p. 4-26, 2012. Disponível em: $<$ http://citrus.uspnet.usp.br/rdg/ojs/index.php/rdg/article/view/117/364>. Acesso em: 21 dez. 2013. 
POURGHASEMI, H. R. et al. Landslide susceptibility mapping using index of entropy and conditional probability models in GIS: Safarood Basin, Iran. CATENA, v. 97, p. 71-84, out. 2012. Disponível em: <http://linkinghub.elsevier.com/retrieve/pii/S034181621200104X>. Acesso em: 5 mar. 2014.

RAFFO, J. G. DA G. O Processo Analítico Hierárquico e seu uso na modelagem do espaço geográfico. Revista do Departamento de Geografia - USP, v. Volume Esp, p. 26-37, 2012. Disponível em: <http://citrus.uspnet.usp.br/rdg/ojs/index.php/rdg/article/view/364/438>. Acesso em: 5 abr. 2014.

SAATY, T. L. The Analytic Hierarchy Process: Planning, Priority Setting, Resource Allocation. New York: McGraw-Hill, 1980.

SANTOS, R. F. DOS. Planejamento Ambiental: teoria e prática. São Paulo: Oficina de Textos, 2004.

SHAHABI, H. et al. Landslide susceptibility mapping at central Zab basin, Iran: A comparison between analytical hierarchy process, frequency ratio and logistic regression models. CATENA, v. 115, p. 5570, abr. 2014. Disponível em: <http://linkinghub.elsevier.com/retrieve/pii/S0341816213002841>. Acesso em: 10 mar. 2014.

SILVA, J. DOS S. V. DA; SANTOS, R. F. DOS. Estratégia metodológica para zoneamento ambiental: A experiência aplicada na Bacia Hidrográfica do Alto Rio Taquari. Campinas: Embrapa Informática Agropecuária, 2011. Disponível em: <http://www.alice.cnptia.embrapa.br/bitstream/doc/913452/1/LivroJVilaInfotecaOut2012.pdf>.

SILVA JUNIOR, C. et al. Mapeamento da suscetibilidade natural a movimentos de massa para a bacia hidrográfica do Rio Anil, São Luís - MA. 2014, Cubatão: COPEC, 2014. p. 118-122. Disponível em: <http://proceedings.copec.org.br/index.php/shewc/article/view/2374>.

SILVA JUNIOR, C. H. L. et al. Uso de Lógica Fuzzy e Processo Analítico Hierárquico - AHP no zoneamento de áreas suscetíveis a deslizamento utilizando o Operador Fuzzy Média Ponderada AHP: o caso da Bacia Hidrográfica do Rio Anil em São Luís - MA. Revista de Geografia, v. 3, n. 2, p. 1-7, 2013. Disponível em: <http://www.ufjf.br/revistageografia/files/2014/07/Artigo-3-RevistaGeografia-Jul2014.pdf>.

SIRAJ, S. et al. PriEsT: an interactive decision support tool to estimate priorities from pairwise comparison judgments. International Transactions in Operational Research, n. 2013, p. 1-19, 18 out. 2013. Disponível em: <http://doi.wiley.com/10.1111/itor.12054>. Acesso em: 7 mar. 2014.

SOUZA, D. F. DE et al. Aplicação do método AHP-Fuzzy. Disponível em: <http://www.pucrs.br/edipucrs/erematsul/comunicacoes/10DEBORAFERRODESOUZA.pdf>. Acesso em: 20 jan. 2013.

VANACÔR, R. N.; ROLIM, S. B. A. Mapeamento da suscetibilidade a deslizamentos usando técnicas de estatística bivariada e sistema de informações geográficas na região nordeste do Rio Grande do Sul. Revista Brasileira de Geomorfologia, v. 13, n. 1, p. 15-28, 2012.

VESTENA, L. R. Mapeamento da susceptibilidade a deslizamentos na bacia hidrográfica do Caeté, Alfredo Wagner/SC. Revista Mercator, v. 9, n. 19, p. 175-190, 30 ago. 2010. Disponível em: <http://www.mercator.ufc.br/index.php/mercator/article/view/361/289>. Acesso em: 14 jun. 2014.

VON-ALTROCK, C. Fuzzy logic and neuroFuzzy applications in busines and finance. New Jersey: Prentice Hall PTR, 1996.

WANG, W. et al. Landslides susceptibility mapping in Guizhou province based on fuzzy theory. Mining Science and Technology (China), v. 19, n. 3, p. 399-404, maio 2009. Disponível em: <http://linkinghub.elsevier.com/retrieve/pii/S1674526409600752>. Acesso em: 27 abr. 2014.

WEB-HIPRE. Servidor do software Web-Hipre. Disponível em: <http://www.hipre.hut.fi/>. Acesso em: 22 jan. 2014.

ZADEH, L. A. Fuzzy sets. Information and Control, v. 8, n. 3, p. 338-353, jun. 1965. Disponível em: <http://linkinghub.elsevier.com/retrieve/pii/S001999586590241X>. Acesso em: 22 jan. 2014. 